\title{
MSVD-Turkish: A Comprehensive Multimodal Dataset for Integrated Vision and Language Research in Turkish
}

\author{
Begum Citamak • Ozan Caglayan • \\ Menekse Kuyu • Erkut Erdem* . \\ Aykut Erdem - Pranava Madhyastha . \\ Lucia Specia
}

Received: date / Accepted: date

\begin{abstract}
Automatic generation of video descriptions in natural language, also called video captioning, aims to understand the visual content of the video and produce a natural language sentence depicting the objects and actions in the scene. This challenging integrated vision and language problem, however, has been predominantly addressed for English. The lack of data and the linguistic properties of other languages limit the success of existing approaches for such languages. In this paper we target Turkish, a morphologically rich and agglutinative language that has very different properties compared to English. To do so, we create the first large scale video captioning dataset for this language by carefully translating the English descriptions of the videos in the MSVD (Microsoft Research Video Description Corpus) dataset into Turkish. In addition to enabling research in video captioning in Turkish, the parallel English-Turkish descriptions also enables the study of the role of video context in (multimodal) machine translation. In our experiments, we build models for both video captioning and multimodal machine translation and investigate the effect of different word segmentation approaches and different neural architectures to better address the properties of Turkish. We hope that the MSVDTurkish dataset and the results reported in this work will lead to better video
\end{abstract}

* Corresponding author.

Begum Citamak · Menekse Kuyu · Erkut Erdem

Department of Computer Engineering, Hacettepe University, Ankara/Turkey

E-mail: \{n16221821, n17132209, aykut, erkut\}@ cs.hacettepe.edu.tr

Aykut Erdem

Department of Computer Engineering, Koç University, Istanbul/Turkey

E-mail: aerdem @ ku.edu.tr

Ozan Caglayan · Pranava Madhyastha · Lucia Specia

Department of Computing, Imperial College London, UK

E-mail: \{o.caglayan, pranava, l.specia\} @ imperial.ac.uk 
captioning and multimodal machine translation models for Turkish and other morphology rich and agglutinative languages.

Keywords Video description dataset · Turkish · Video captioning · Video understanding $\cdot$ Neural machine translation $\cdot$ Multimodal machine translation

\section{Introduction}

Recent developments in computer vision (CV) and natural language processing (NLP) have led to a surge of new problems which lie at the intersection of these two fields, creating a new area of research in general called integrated vision and language (iVL). Video captioning is one of the important problems in iVL research, which has gained significant attention in both the CV and NLP communities. It aims at understanding the visual content of a given video clip and contextually generating a natural language description of this clip.

Although considerable literature has revolved around this challenging task in recent years, all existing work is monolingual that it has mainly focused on the English language. Hence, whether or not the state-of-the-art video captioning methods can be effectively adapted to languages other than English, especially for low-resource languages, remains an open problem. Moreover, linguistic differences between English and other languages, particularly the ones that are morphologically richer than English, introduce new challenges that need to be addressed. Before these questions can be answered, however, we require video datasets containing descriptions from languages other than English to further enable iVL research.

As a first step towards this direction, in this paper, we extend the MSVD (Microsoft Research Video Description Corpus) (Chen and Dolan, 2011) dataset and introduce a new multilingual dataset that we call MSVD-Turkish which contains approximately $2 \mathrm{k}$ video clips and a total of $80 \mathrm{k}$ Turkish video descriptions. In particular, we collect these Turkish descriptions by manually translating the original English video descriptions from MSVD into Turkish. As compared to the original English descriptions, Turkish descriptions have a larger vocabulary size and more importantly reflects the highly inflected and highly agglutinative nature of Turkish.

We demonstrate the multilingual, multimodal capabilities of the proposed MSVD-Turkish dataset, by exploring two distinct iVL tasks shown in Figure 1. namely video captioning and multimodal machine translation, but with a special focus on Turkish. As far as we are aware of, this is the first to investigate generating Turkish descriptions depicting visual content of videos. To this end, we analyse different segmentation strategies for Turkish. Additionally, we explore multimodal machine translation as the second task on MSVD-Turkish where we examine the use of supplementary visual cues within videos to potentially improve the translation quality. Our primary contributions in this paper are:

- To foster the research in the multilingual, multimodal language generation, we collect a new large scale dataset called MSVD-Turkish by translating 

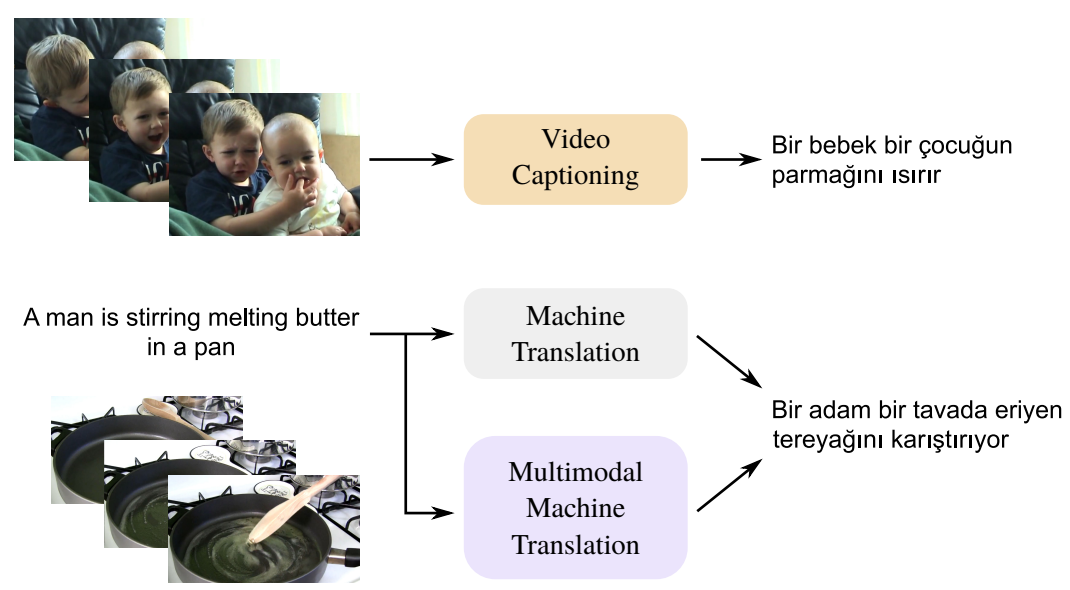

Fig. 1 The depiction of the video captioning and machine translation tasks on the MSVDTurkish Dataset.

the English descriptions of the videos from the well-known MSVD dataset into Turkish.

- We investigate the performance of several (multimodal) machine translation and video captioning models on the proposed MSVD-Turkish dataset.

- To address the rich and agglutinative morphology of Turkish, we explore different word segmentation approaches in Turkish.

The paper is organised as follows: In Section 2 , we briefly review the stateof-the-art in multimodal machine translation and video captioning. In Section 3, we introduce the MSVD-Turkish dataset, examine our data collection strategy and provide some statistics regarding the dataset. We introduce the details regarding the visual and textual representations considered in our machine translation and video captioning models in Section 4, and describe the models themselves in Section 5 In Section 6, we present our experimental results and discuss our findings and finally, we provide a summary of our work and discuss possible future research directions in Section 7

\section{Related Work}

In the following, we review the efforts towards two related tasks within the integrated vision and language research, namely multimodal machine translation and video captioning.

\subsection{Multimodal Machine Translation}

The predominant approaches in state-of-the-art in machine translation (MT) use neural models (NMT) which consist of an encoder to map a given sentence 
into a latent representation, and a decoder to map this representation into a translation in the target language (Sutskever et al., 2014, Cho et al., 2014, Bahdanau et al., 2015; Vaswani et al., 2017). NMT models are trained with maximum likelihood estimation (MLE), i.e. the training objective maximises the likelihood of source-target training pairs.

The success of such approaches has led to a rising interest in more sophisticated NMT architectures that can handle multiple input/output modalities, a framework often referred to as multimodal machine translation (MMT). MMT seeks to enhance the translation quality by taking into account visual (Specia et al., 2016, Elliott et al. 2017; Barrault et al., 2018) or speech modality (Sulubacak et al. (2019). The prominent end-to-end approaches to MMT with visual information can be divided into two main categories:

1. Multimodal attention extends the classical attention mechanism (Bahdanau et al. 2015) applied on top of the textual representations, with a spatial one (Xu et al., 2015) applied to convolutional feature maps. Specifically, Caglayan et al. (2016a) explore a shared attention across the modalities while Calixto et al. (2016) experiment with dedicated attention. Caglayan et al. (2016b) later propose several variants where the level of parameter sharing across modality attentions is configurable. In all these models, the outputs of attention mechanisms are simply fused together with addition or concatenation. Libovický and Helcl (2017) replace this step with another attention layer which could in theory, selectively integrate information coming from different modalities. Huang et al. (2016) do not implement a fully multimodal attention in the decoder but enriches the source word embedding sequence with visual feature vectors, with the hope that the decoder attention will learn to pay attention to visual feature vectors when needed.

2. Simple conditioning makes use of non-spatial features such as the fullyconnected (FC) layer features for VGG-style (Simonyan and Zisserman 2014) and global average pooled features for ResNet-style (He et al. | 2016) networks. Specifically, a single feature vector per image is used to condition arbitrary layers in the network, with the objective of learning grounded textual representations. The visual conditioning is often performed through (i) initializing the hidden state of the recurrent encoders and/or decoders with the visual features (Calixto and Liu, 2017), (ii) multiplicative interactions of source and/or target embeddings with the visual features (Caglayan et al. 2017a), and (iii) the use of auxiliary training objectives such as the Imagination architecture (Elliott and Kádár, 2017) which tries to reconstruct the visual features from the textual encoder states.

\subsection{Video Captioning}

Video captioning aims at generating a single sentence description from a short video clip summarising the actors and actions depicted in the clip. It involves 
unique challenges over image captioning, since it additionally requires analyzing the temporal evolvement of concepts and their relationships. The methods proposed for this task can be, in general, categorised into three classes (for a more thorough review, please refer to (Aafaq et al., 2019)):

1. Classical video captioning approaches include the early works that integrate the traditional computer vision and natural language processing techniques (Kojima et al., 2012, Hakeem et al., 2004; Barbu et al., 2012, Hanckmann et al., 2012; Krishnamoorthy et al., 2013, Guadarrama et al. 2013: Thomason et al. 2014). These methods commonly generate description of a clip in two phases. In the first phase, they generally detect the most important objects, recognise their actions and extract object-object interactions along with the scene information. The second phase employs these extracted visual entities and some rule-based, pre-defined sentence templates to construct video descriptions. This strategy, while generates grammatically sound sentences, the sentences lack naturalness and more importantly become too constrained for open-domain videos.

2. Statistical video captioning methods such as (Rohrbach et al., 2013) have been proposed to fill in this gap by additionally taking into account some statistical cues while generating a natural language description of a given input video, and thus provides more accurate and more natural depictions as compared to the classical approaches.

3. Deep video captioning approaches which are specifically motivated from the recent neural machine translation models. They all consider two sequential stages which are realised with an encoder-decoder architecture. The basic difference between these deep learning models and the first two groups of works lies in how they represent the visual content. While the earlier approaches employ recognition and detection methods to extract a set of word tokens, deep models represent the video in terms of a vector representation, either with a fixed or dynamic embedding. Deep learning based video captioning models can be categorised into further groups by their encoder-decoder structure and by their learning methodology. For instance, the most common model architecture (Donahue et al., 2015, Venugopalan et al. 2015: Yao et al. 2015) employs convolutional neural networks to extract visual content in the encoding stage and a recurrent neural network in the decoding phase to perform the video-driven sentence generation. Some other works (Srivastava et al., 2015; Yu et al., 2016)) extend this structure by considering recurrent neural networks in both encoding and decoding stages. The final group of studies includes reinforcement learning based video captioning models (Wang et al., 2018, Chen et al., 2018).

\section{MSVD-Turkish Dataset}

Existing datasets for video captioning typically contain short video clips (a few seconds in duration) and descriptions depicting the content of videos in a natural language. The early examples such as MPII Cooking (Rohrbach et al. 
Table 1 Statistics of Video Captioning Datasets

\begin{tabular}{|c|c|c|c|c|c|c|c|c|}
\hline Dataset & Domain & Classes & Videos & Avg len & Clips & Sents & Words & Vocab \\
\hline MPII Cooking & cooking & 65 & 44 & $600 \mathrm{sec}$ & - & 5,609 & - & - \\
\hline YouCook & cooking & 6 & 88 & - & - & 2,688 & 42,457 & 2,711 \\
\hline TACoS & cooking & 26 & 127 & $360 \mathrm{sec}$ & 7,206 & 18,227 & 146,771 & 28,292 \\
\hline TACos-MLevel & cooking & 1 & 185 & $360 \mathrm{sec}$ & 14,105 & 52,593 & 2,000 & - \\
\hline MPII-MD & movie & - & 94 & $3.9 \mathrm{sec}$ & 68,337 & 68,375 & 653,467 & 24,549 \\
\hline M-VAD & movie & - & 92 & $6.2 \mathrm{sec}$ & 48,986 & 55,904 & 519,933 & 17,609 \\
\hline MSR-VTT & open & 20 & 7,180 & $20 \mathrm{sec}$ & $10 \mathrm{k}$ & $200 \mathrm{k}$ & $1,856,523$ & 29,316 \\
\hline Charades & open & 157 & 9,848 & $30 \mathrm{sec}$ & - & 27,847 & - & - \\
\hline VTW & open & - & 18,100 & $90 \mathrm{sec}$ & - & 44,613 & - & - \\
\hline YouCook II & cooking & 89 & 2,000 & $316 \mathrm{sec}$ & $15.4 \mathrm{k}$ & $15.4 \mathrm{k}$ & - & 2,600 \\
\hline ActyNet Cap & open & - & $20 \mathrm{k}$ & $180 \mathrm{sec}$ & - & $100 \mathrm{k}$ & $1,348,000$ & - \\
\hline ANet-Entities & social media & - & 14,281 & $180 \mathrm{sec}$ & $52 \mathrm{k}$ & - & - & - \\
\hline VideoStory & social media & - & $20 \mathrm{k}$ & - & $123 \mathrm{k}$ & $123 \mathrm{k}$ & - & - \\
\hline VATEX & open & 600 & $41.3 \mathrm{k}$ & - & $41.3 \mathrm{k}$ & $826 \mathrm{k}$ & - & - \\
\hline MSVD & open & 218 & 1970 & $10 \mathrm{sec}$ & 1,970 & 80,827 & 567,874 & 12,592 \\
\hline MSVD-Turkish & open & 218 & 1970 & $10 \mathrm{sec}$ & 1,970 & 80,676 & 432,250 & 18,312 \\
\hline
\end{tabular}

2012), YouCook (Das et al., 2013), TACoS (Regneri et al., 2013), TACoS Multi-Level (Senina et al., 2014), and YouCook II (Zhou et al., 2017) include videos about everyday actions, which were usually collected from the video sharing sites such as YouTube by querying keywords related to cooking. On the other hand, more recent datasets such as MSVD (Chen and Dolan, 2011), M-VAD (Torabi et al., 2015), MPII-Movie Description (MPII-MD) (Rohrbach et al. 2015), TGIF (Li et al., 2016), MSR-VTT (Xu et al., 2016), VTW (Zeng et al. 2016), Charades (Sigurdsson et al., 2016), LSMDC (Rohrbach et al. 2017), ActyNet-Cap (Krishna et al., 2017), ANet-Entities (Zhou et al., 2019), and VideoStory (Gella et al. . 2018) are open-domain datasets. M-VAD, MPII$\mathrm{MD}$, and LSMDC datasets differ from the others in that they contain movie clips descriptions constructed by professionals for descriptive video service purposes. In recent years, with the increase in the use of social media platforms, social media has become a major source of data, and ANet-Entities (Zhou et al., 2019) and VideoStory (Gella et al. 2018) datasets include videos shared in these mediums. Table 1 summarises the characteristics of these datasets in detail.

It is important to mention that all these datasets are monolingual and contain only English descriptions. The only exception is the recently proposed VATEX dataset (Wang et al., 2019), which has both Chinese and English descriptions for each video clip. Even for image captioning which has been studied more extensively than video captioning, multilingual datasets are scarce. There exist only a few datasets such as (i) the TasvirEt dataset (Unal et al., 2016) which extends the original Flickr8k dataset with two crowdsourced Turkish descriptions per image, (ii) the STAIR dataset (Yoshikawa et al., 2017) which 
provides five crowd-sourced Japanese descriptions for 164,062 MSCOCO (Lin et al. 2014) images. Similarly, the image-based MMT task requires a multimodal dataset with images and their (translated) descriptions in at least two languages. The well-known Multi30k dataset (Elliott et al., 2016) fulfilled this requirement by augmenting the popular image captioning dataset Flickr30k (Plummer et al. 2015), with German, French and Czech descriptions that are direct translations of the original Flickr30k English descriptions. Multi30k is so far the only dataset which provides actual translations aligned to images, rather than independent descriptions as in TasvirEt and STAIR datasets.

In this study, we aim to contribute to this new area of research, multilingual video captioning, by collecting a large video dataset consisting of videos and their English and Turkish descriptions. We believe that selecting Turkish will fill an important gap on the analysis of morphologically-rich and low-resource languages in the video captioning literature. We name our dataset as MSVDTurkish, after the MSVD dataset. Since MSVD-Turkish has parallel TurkishEnglish sentences, it can be used not only for video captioning task but also for multimodal machine translation 1

In the data collection phase, we first translated the English captions into Turkish with the free Google Translate API. The main drawback of using such an automated machine translation system is that the generated translations could be of low-quality. We observed that in some of the translations, suffixes were incorrectly attached or they were completely missing. Moreover, there were some translation issues regarding ambiguous words. For this reason, we gathered a team of two M.Sc. students who are Turkish-English bilingual speakers with some experience on image captioning. They checked the automatically translated sentences for obvious errors and made the necessary corrections accordingly. During this, we noticed that some English sentences in the original MSVD dataset are very noisy - some are not even in English. We left those sentences out and did not translate them into Turkish. Figure 2 depicts an example video clip with the original English descriptions from MSVD and their translations into Turkish as provided in the resulting MSVD-Turkish dataset.

As can be seen in Table 1, the MSVD dataset contains a total of 1,970 video clips collected from YouTube, which about 10 seconds in length, with an average of 41 descriptions per clip. The training, validation and test sets from MSVD are preserved and they contain 1200, 100 and 670 videos, respectively. The number of descriptions in MSVD-Turkish is slightly lower due to the removal of noisy captions in the original MSVD. We note that there is a substantial increase in the vocabulary size $(45 \%)$ for Turkish, which reflects the sparsity induced by its rich morphology. Moreover, we observe that the average number of words in the descriptions is decreased in the translations, which is expected to some extent due to the agglutinating nature of Turkish in that individual words can represent multiword expressions in English.

1 We make our dataset publicly available at https://hucvl.github.io/MSVD-Turkish/ 


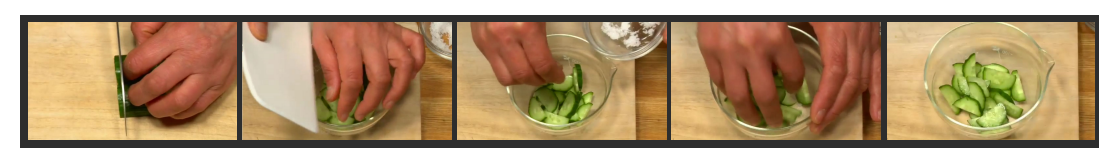

MSVD Descriptions

a woman is cutting a vegetable a man cuts a piece of zucchini in half and slices it thinly a person is slicing cucumbers

someone sliced a cucumber or a zucchini into small pieces a person is slicing a cucumber into pieces

a chef is slicing a vegetable

a person is slicing a cucumber

a woman is slicing vegetables

a woman is slicing a cucumber

a person is slicing cucumber with a knife
MSVD-Turkish Descriptions

bir kadın bir sebze kesiyor

bir adam bir parça kabağı ikiye keser ve ince dilimler bir kişi salatalık dilimliyor

birisi küçük parçalar halinde bir salatalık ya da bir kabak dilimlenmiş

bir kişi bir salatalığı parçalara ayırıyor

bir şef bir sebze dilimliyor

bir kişi bir salatalık dilimliyor

bir adam sebze dilimliyor

bir kadın bir salatalık dilimliyor

bir kişi bir bıçakla salatalık dilimliyor

Fig. 2 A sample clip and its English captions with the corresponding Turkish translations from the MSVD-Turkish dataset.

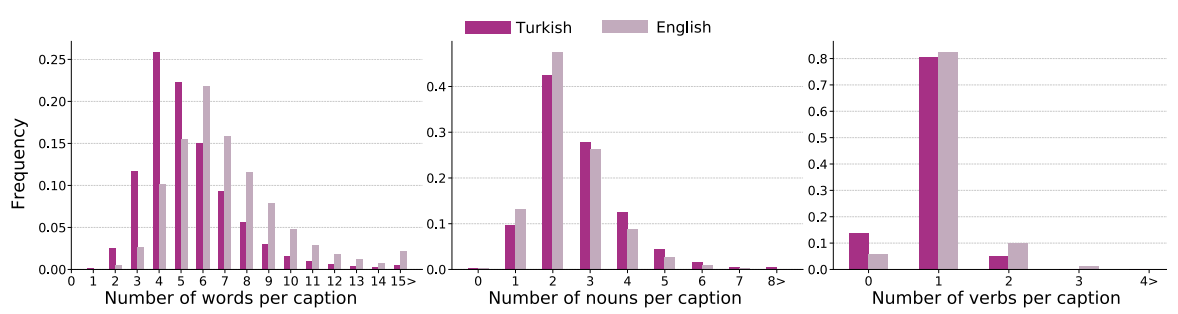

Fig. 3 Word, noun and verb count distributions across MSVD and MSVD-Turkish datasets.

Figure 3 presents a more comprehensive comparison between the MSVD and MSVD-Turkish datasets. Here, we utilised the NLTK toolkit (Loper and Bird, 2002) for English and the Zemberek toolkit (Akin and Akin, 2007) for Turkish to compute unique word, noun and verb counts in the descriptions. Linguistically, Turkish descriptions are shorter but they also contain more nouns and verbs, as compared to the case in English captions. Taking into account the differences in these distributions, we can say that the same expressions have different structures in Turkish and English.

\section{Modality Representations}

Both the video captioning and machine translation tasks can be cast as sequence to sequence $(\mathrm{S} 2 \mathrm{~S})$ problems, and they involve processing of visual and/or textual information. In the following, we briefly explain our choices for video and textual representations.

Visual representations We represent each video clip with a fixed-length vector by using an ImageNet pre-trained VGG16 Simonyan and Zisserman (2014) network. In particular, we sample 30 equidistant frames from each video and feed them to VGG16 to extract 4096-dimensional FC7 feature vectors. The final video representation that will be used by the video captioning models is this temporal feature matrix of size $30 \times 4096$. 
Textual representations In addition to the default setting of using words for both languages, we conduct several experiments using two widely-known unsupervised segmentation techniques. The motivation behind these experiments is to understand what kind of token representation is ideal for video captioning and machine translation into Turkish, an agglutinative language with rich morphology.

The first segmentation approach makes use of the so-called "byte-pair encoding (BPE)" algorithm, which proceeds by deconstructing sentences to characters and then works out in a bottom-up manner to recursively merge frequent byte pairs altogether (Sennrich et al., 2016). The final "open vocabulary" is biased towards keeping frequent words intact while splitting out rare words into frequent subwords. The main hyperparameter of the BPE algorithm determines how many merge operations will be done during the learning step, which approximately reflects the final vocabulary size. In contrast to the BPE algorithm which is deterministic, SentencePiece (SPM) employs a unigram language modeling approach to maximize the likelihood of a given corpus with the probability of each sentence defined as the sum of its candidate segmentations (Kudo and Richardson, 2018). Overall, this yields a probabilistic mixture model from which it is possible to sample arbitrarily many multi-level segmentations (characters, subwords and words) for a given sentence. SPM can also be applied to non-tokenized sentences, removing the necessity of using language-dependent tokenization and detokenization pipelines.

\section{Tasks and Models}

In this section, we present two tasks exploring the proposed bilingual MSVD dataset where the common objective is to generate natural language sentences in Turkish. We first start by describing the sequence-to-sequence framework within the context of monolingual and multimodal machine translation. We then present the video captioning approaches that we follow, which can be considered as extensions to the S2S framework. We note that some design choices and hyperparameters are different between the neural architectures and the video captioning and MMT tasks because they were empirically selected for each architecture and task.

\subsection{Multimodal Machine Translation}

In what follows, we introduce our recurrent and transformer-based NMT models and their multimodal counterparts. To represent the words in the sentences, we experiment with the segmentation approaches previously mentioned in Section 4. To achieve multimodality, we provide the 4096-dimensional frame representations extracted from the pre-trained CNN (Section 4) as a secondary input modality along the English sentences (Figure 4). Since videos are temporally represented by a sequence of frame features, we employ the multimodal 


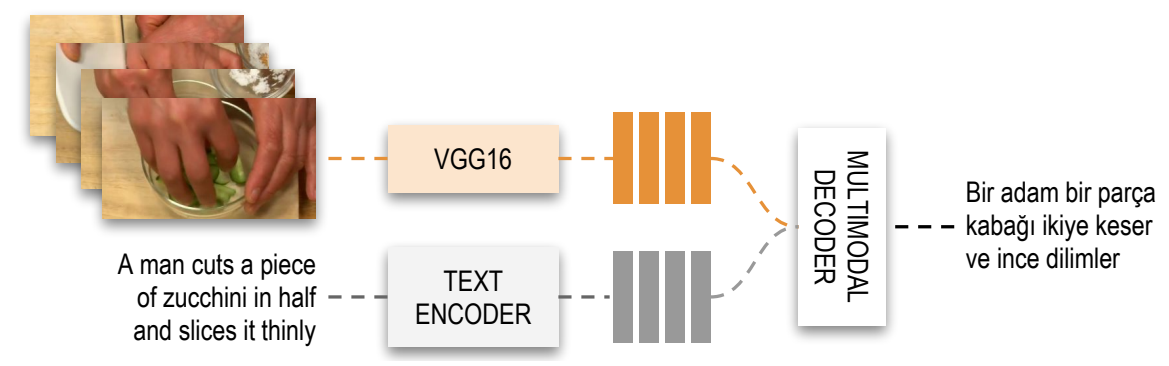

Fig. 4 Multimodal machine translation decoder on MSVD-Turkish.

attention approach described in Section 2.1. Model-specific details will be given in the respective subsections.

\subsubsection{Recurrent Model}

We follow the attentive encoder-decoder approach (Bahdanau et al., 2015) for the recurrent models. The attention mechanism is crucial to obtain state-ofthe-art results in neural machine translation (NMT). This mechanism avoids encoding the whole source sentence into a single vector as in Sutskever et al. (2014) by looking to the latent encodings of the source sentence at each timestep of the decoder. In other words, the decoder is conditioned on a different representation of the source sentence (namely the context $c_{t}$ ) when generating target words, rather than reusing the same fixed-size encoding vector. The model thus estimates the probability of a target token $y_{t}$ by conditioning on the previous target token $y_{t-1}$ and the dynamic context $c_{t}$ i.e. $P\left(y_{t} \mid y_{t-1}, c_{t}\right)$.

Architecture Our recurrent baseline is composed of two bidirectional GRU (Cho et al. 2014) layers in the encoder. The Turkish decoder follows the Conditional GRU (CGRU) design (Sennrich et al., 2017) where the attention mechanism operates between two GRU layers. The input and output embeddings of the decoder are tied for parameter efficiency (Press and Wolf 2017; Inan et al., 2016). The hidden dimensions of encoders, decoders and the attention mechanism are globally set to 320 while the source and target embeddings are 200-dimensional. Dropout (Srivastava et al., 2014) is employed at three places, namely, on top of the source embeddings $(p=0.4)$, encoder outputs $(p=0.5)$ and before the softmax layer $(p=0.5)$. We train the models using the open-source sequence-to-sequence toolkit nmtpytorch (Caglayan et al., 2017b).

Multimodality We first experiment with the dedicated multimodal attention mechanism with concatenation (Caglayan et al. 2016b). In this setup, the 4096-dimensional VGG features are projected to 640 i.e. the dimension of textual encoder states. We also experiment with simple conditioning, namely by initialising the encoders and the decoders by the max-pooled VGG feature vector. 
Training We evaluate model performance after each epoch by computing the BLEU (Papineni et al. 2002) score for the validation set translations. The learning rate is halved if the performance does not improve for two consecutive epochs. After five consecutive epochs with no improvement, the training is stopped. At test time, the translations are decoded using the best model checkpoint, with beam size set to 12 .

\subsubsection{Transformer Model}

Transformer-based NMT (Vaswani et al., 2017) are feed-forward architectures which extend the idea of attention and avoid the need for recurrent layers. This has mainly two advantages: (i) it accommodates for more layers (depth) as the gradients will flow more easily than recurrent NMTs, (ii) the removal of sequential dependence between hidden states allows parallelised training. The expressiveness of recurrence is replaced with self-attention layers which takes into account all hidden representations at a given depth. Transformer NMTs are currently state of the art, especially in large-scale NMT setups.

Architecture $\mathcal{G}$ Training Since the dataset is relatively small, we use the base Transformer model with 6 encoders and 6 decoder layers, each having 4 attention heads. The model and feed-forward dimensions are set to 256 and 1024, respectively. Label smoothing with $\epsilon=0.1$ is applied to the crossentropy loss. The dropout rate is fixed as 0.3 . We use the open-source fairseq toolkit (Ott et al. 2019) for training the models. The models are trained for a maximum of 50 epochs, and the test set translations are generated with beam size 12, using the checkpoint that achieved the lowest validation loss during training.

\subsection{Video Captioning}

Similar to NMT models, deep approaches to video captioning also employ encoder-decoder architectures. While the encoder module takes the individual frame representations as input and encodes them into a feature representation, the decoder generates a natural language description of the video by considering the encoded visual information. In this study, we test two popular types of network architectures, namely a recurrent one which uses the LSTM (Hochreiter and Schmidhuber, 1997) variant and a Transformer (Vaswani et al., 2017). As we mentioned in Section 4 4 we utilise the ImageNet pre-trained VGG16 CNN to encode the video frames, and as for the textual representation, we investigate different word segmentation strategies using SPM and BPE algorithms.

\subsubsection{Recurrent Model}

Architecture For our recurrent video captioning model, we adapt the architecture proposed by Venugopalan et al. (2015) in which the encoder and 


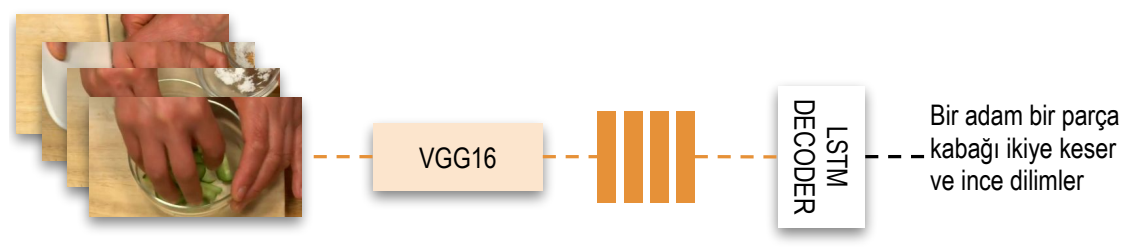

Fig. 5 Architecture of the LSTM-based video captioning model.

the decoder are implemented with two separate LSTM networks (Figure 5). The encoder computes a sequence of hidden states by sequentially processing the 4096-dimensional VGG16 features, extracted from the uniformly sampled video frames. The decoder module, then, takes the final hidden state of the encoder, and accordingly outputs a sequence of tokens as the predicted video caption. There is no attention mechanism involved in this model. Both the encoder and decoder LSTM networks have 500 hidden units.

Training We use ADAM (Kingma and Ba, 2014) as the optimiser and set the initial learning rate and batch size to 0.0004 and 32 , respectively. We choose the models by using early stopping. In particular, we take into account the validation loss values to decide for the checkpoint that will be used to generate Turkish descriptions at inference time.

\subsubsection{Transformer Model}

Architecture Our Transformer-based video captioning model is built upon the base Transformer model (Vaswani et al., 2017). In the encoder, we first consider a linear transformation layer to project the extracted VGG16 features to 512. We then treat these transformed features as our visual tokens, and consider positional encodings to preserve temporal information of the frames. The decoder module is responsible for generating a description conditioned on the input video frames encoded by the encoder. Figure 6 shows an illustration of our Transformer-based video captioning model.

Training We train the models using tensor2tensor toolkit Vaswani et al. (2018). We use the base Transformer model containing 3 encoder and 3 decoder layers, each with 8 attention heads, since the dataset contains few video samples. During training, we employ the cross entropy loss with label smoothing $(\epsilon=0.1)$ and a batch size of 1024 . The dropout rate is fixed as 0.1 . The model parameters are optimised using ADAM by setting the initial learning rate to 0.0005 . We employ approximate BLEU score for early stopping, and at test time, descriptions are obtained by using beam search with a beam size of 4 . 


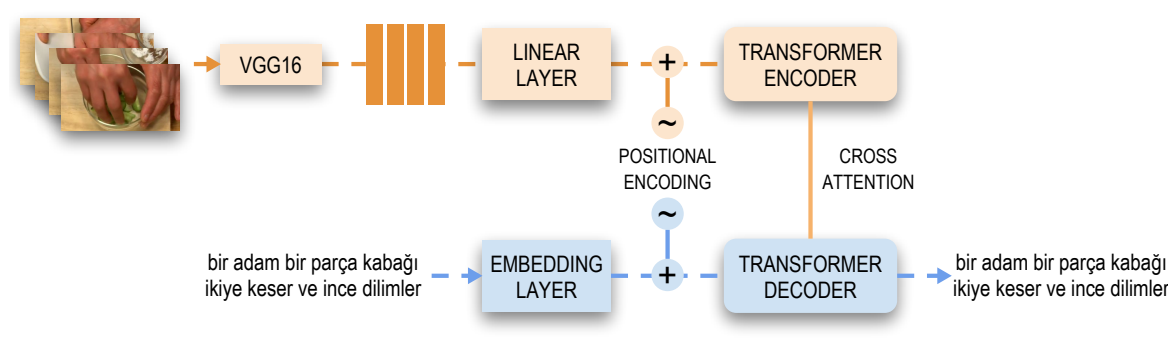

Fig. 6 Illustrative architecture of the Transformer-based video captioning model.

\section{Experimental Results}

In this section we present quantitative and qualitative results for the previously described machine translation and video captioning experiments. But before doing so, we provide the results regarding the choice of sentence segmentation strategies based on experiments with the translation as a guidance (Section 4).

\subsection{Machine Translation}

We first focus our attention on the word segmentation problem. For that, we use of the parallel captions from the MSVD-Turkish dataset and learn separate English and Turkish segmentation models using BPE and SPM approaches. We explore four settings where the size of the final vocabularies would be approximately $3 \mathrm{~K}$ and $6 \mathrm{~K}$ tokens. We train three RNN NMTs per each segmentation setup and report the mean and the standard deviation of test set BLEU scores. We also train a non-segmented word $\rightarrow$ word baseline for comparison. Table 2 presents the results for these experiments. We observe that in almost all cases SPM outperforms BPE, with the average gap being 0.5 BLEU in favor of SPM. Surprisingly, the best average performance 36.2 is obtained when words are used without segmentation.

Pre-training segmentation models. These results do not favor any of the proposed segmentation approaches. We posit that this may be because of limitations of the segmentation models themselves. Therefore, we directed our attention to pre-training Turkish BPE and SPM models on a large external corpus instead of the small set of MSVD-Turkish captions. This way, we expect to learn slightly more linguistically sound segmentation models. For this purpose, we used a snapshot (2019-07-20) of the official Turkish Wikipedia dump, pre-processed it ${ }^{2}$ and ended up with $955 \mathrm{~K}$ Turkish sentences. In order

\footnotetext{
2 Pre-processing consists of lowercasing, length filtering with minimum token count set to 5 , punctuation removal and deduplication.
} 
Table 2 BLEU comparison of English and Turkish segmentation choices with RNN NMT. A word $\rightarrow$ word baseline is also provided for comparison purposes. The segmentation models are learned on the training set of the MSVD dataset.

\begin{tabular}{rcccccc}
\hline & Word $\rightarrow$ Word & $\mathbf{3 K} \rightarrow \mathbf{3 K}$ & $\mathbf{3 K} \rightarrow \mathbf{6 K}$ & $\mathbf{6 K} \rightarrow \mathbf{3 K}$ & $\mathbf{6 K} \rightarrow \mathbf{6 K}$ & $\mathbf{A v g}$ \\
\hline Words & $\mathbf{3 6 . 2} \pm 0.3$ & - & - & - & - & - \\
$\mathrm{BPE}$ & - & $35.4 \pm 0.4$ & $34.5 \pm 0.7$ & $36.1 \pm 0.3$ & $35.1 \pm 0.6$ & 35.3 \\
$\mathrm{SPM}$ & - & $35.9 \pm 0.4$ & $35.9 \pm 0.3$ & $36.0 \pm 0.2$ & $35.3 \pm 0.2$ & 35.8 \\
\hline
\end{tabular}

Table 3 BLEU comparison of Turkish Wikipedia pre-trained segmentation models for RNN and Transformer NMT models. The English vocabulary is fixed to word units and contains 9,321 words in total. The corresponding Turkish vocabulary sizes are given in parentheses.

\begin{tabular}{rccc}
\hline & $\rightarrow$ Word (13400) & $\rightarrow$ BPE30K (8400) & $\rightarrow$ SPM30K (7900) \\
\hline Transformer & $35.8 \pm 0.2$ & $36.5 \pm 0.2$ & $36.7 \pm 0.1$ \\
RNN & $36.2 \pm 0.3$ & $36.8 \pm 0.3$ & $\mathbf{3 7 . 0} \pm 0.6$ \\
\hline
\end{tabular}

Table 4 Multimodal machine translation results on MSVD-Turkish. The Turkish vocabulary uses SPM30K learned on Wikipedia and English vocabulary consists of words.

\begin{tabular}{lrc}
\hline Model & \# Params & BLEU \\
\hline RNN & $8.3 \mathrm{M}$ & $\mathbf{3 7 . 0} \pm 0.6$ \\
+ Multimodal Attention & $11.3 \mathrm{M}$ & $36.5 \pm 0.1$ \\
+ Enc-Dec Initialisation & $9.4 \mathrm{M}$ & $36.7 \pm 0.1$ \\
\hline
\end{tabular}

to focus on the effect of segmentation in Turkish, we fix the segmentation of the English vocabulary to word units and explore only target language segmentation strategies. Table 3 presents the results for both RNN NMT and Transformer-based NMT. We see that the SPM30K model trained on Wikipedia performs consistently better than the others for both types of architectures. This result clearly shows the benefit of segmentation by using a large external corpus for the morphologically-rich Turkish language. We also notice that for the MSVD-Turkish dataset, the recurrent CGRU architecture slightly outperforms the Transformer models in every setting.

Multimodal Machine Translation We now fix the choice of segmentation to words for English and to SPM30K for Turkish, and proceed with the multimodal machine translation results. Here, we limit the experiments to recurrent MMTs since the monomodal results did not reveal any advantages for Transformer NMT in terms of performance (Table 3). Table 4 shows the results for the multimodal machine translation experiments. We observe that none of the multimodal architectures can surpas ${ }^{3}$ the strong RNN baseline on average. This could be because of the multimodal fusion strategy employed here and could be improved by a more sophisticated multimodal design.

\footnotetext{
3 It should be noted we reused the hyper-parameters from the NMT experiments and did not conduct a hyper-parameter search for MMTs.
} 
Table 5 Quantitative comparison of the LSTM and Transformer-based Turkish video captioning models in terms of BLEU, METEOR, ROUGE-L and CIDEr metrics: word, BPE, SPM-based scores and the number of trainable parameters are reported, with bold-face denoting the best performance for each architecture.

\begin{tabular}{|c|c|c|c|c|c|c|}
\hline & Vocab & BLEU & METEOR & ROUGE-L & CIDEr & \# Params \\
\hline \multirow{3}{*}{ 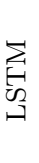 } & Word & $23.2 \pm 1.6$ & $23.4 \pm 0.5$ & $55.3 \pm 1.3$ & $25.4 \pm 1.7$ & $25.2 \mathrm{M}$ \\
\hline & BPE30K & $22.7 \pm 2.6$ & $\mathbf{2 4 . 7} \pm 1.4$ & $53.8 \pm 1.8$ & $\mathbf{2 5 . 4} \pm 1.8$ & $17.1 \mathrm{M}$ \\
\hline & SPM30K & $22.1 \pm 1.0$ & $23.9 \pm 0.3$ & $54.8 \pm 0.4$ & $24.6 \pm 1.2$ & $16.3 \mathrm{M}$ \\
\hline \multirow{3}{*}{ 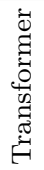 } & Word & $\mathbf{2 4 . 1} \pm 0.2$ & $26.1 \pm 0.1$ & $58.5 \pm 0.2$ & $38.3 \pm 0.2$ & $24.1 \mathrm{M}$ \\
\hline & BPE30K & $23.8 \pm 0.1$ & $26.7 \pm 0.0$ & $58.4 \pm 0.2$ & $40.0 \pm 0.6$ & $21.1 \mathrm{M}$ \\
\hline & SPM30K & $23.9 \pm 0.0$ & $\mathbf{2 6 . 9} \pm 0.0$ & $\mathbf{5 9 . 1} \pm 0.1$ & $38.2 \pm 0.1$ & $20.7 \mathrm{M}$ \\
\hline
\end{tabular}

\subsection{Video Captioning}

In our quantitative analysis, we employ four commonly used evaluation metrics in captioning, namely BLEU (Papineni et al., 2002), METEOR (Denkowski and Lavie, 2014), ROUGE (Lin, 2004) and CIDEr (Vedantam et al., 2015). The scores are computed with coco-eval toolkit (Lin et al., 2014). In our experiments, we train each model five times with different random seeds and report the average performances over all the runs and the corresponding standard deviations.

As stated earlier, we experiment with both LSTM and Transformer-based architectures and analyze three different segmentation strategies, namely word, BPE and SPM level segmentations. As can be seen from the results presented in Table 5. Transformer-based models generate more accurate descriptions than the LSTM-based models. Moreover, switching from words to subword units extracted using SPM or BPE improves the performance in general except BLEU. Additionally, SPM and BPE based models have less number of trainable parameters than word-based models. We note that the results in Table 5 are not directly comparable to the ones in Table 4 since for the machine translation experiments we consider a single reference, whereas for the video captioning experiments we consider all available references. This follows from previous work in these areas, where for MT every pair of source-reference segment is treated as an additional instance.

In Figure 7, we show some qualitative results of the proposed LSTM and Transformer-based Turkish captioning models and monomodal and multimodal recurrent MT models. In the top row, we show some sample results from our models where they give satisfactory translations and generations. On the other hand, we provide some corner cases in the bottom row, where the proposed models produce semantically and/or grammatically incorrect outputs. This demonstrates that there are still some open challenges and room for further research. 
(A)

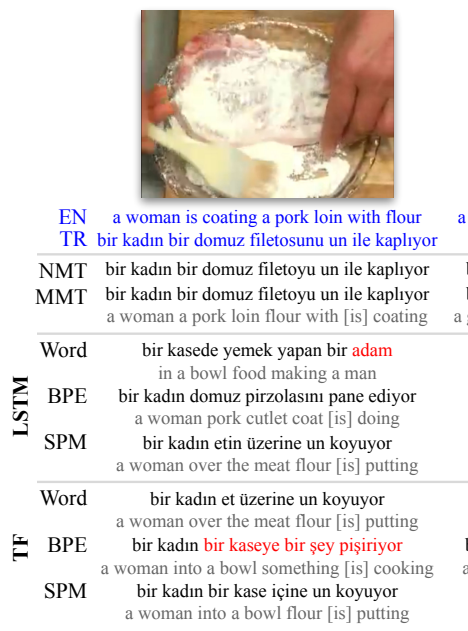

(D)
(B)

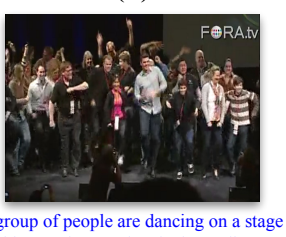

group of people are dancing on a stage

bir grup insan sahnede dans ediyor

bir grup insan bir sahnede dans ediyor

bir grup insan bir sahnede dans ediyor
(C)

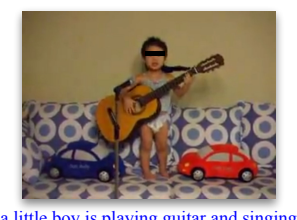

and singing

küçuk bir çocuk gitar çalıyor ve șarkı söylüyor

küçük bir çocuk gitar çalıyor ve şarkı söylüyor küçük bir çocuk gitar çalıyor ve şarkı söylüyo little child guitar [is] playing and song [is] singing $\begin{array}{cc}\text { dans eden bir grup } & \text { bir kı bir gitar çalyor } \\ \text { dance doing a group } & \text { a girl a guitar [is] playing } \\ \text { dans eden bir grup } & \text { bir kız müzik çaliyor }\end{array}$ dans eden bir grup $\quad$ a girl music [is] playing bir grup insan dans ediyor $\begin{array}{ll}\text { bir grup insan dans ediyor } & \text { bir çocuk bir enstrüman çalyor } \\ \text { g group people [are] dance doing } & \text { a child an instrument [is] playin }\end{array}$ bir çocuk bir enstrüman çalıyor $\begin{array}{ll}\text { bir grup dans ediyor } & \text { bir kiz gitar çallyor }\end{array}$ a group dance [is] doing a girl guitar [is] playing bir grup erkek bir sahnede dans ediyor bir kız müzik çalıyor a girl music [is] playing bir kız bir flüt çalıyor a girl a flute [is] playing bir grup insan dans ediyor a group people dance $[$ is] doing

(F)

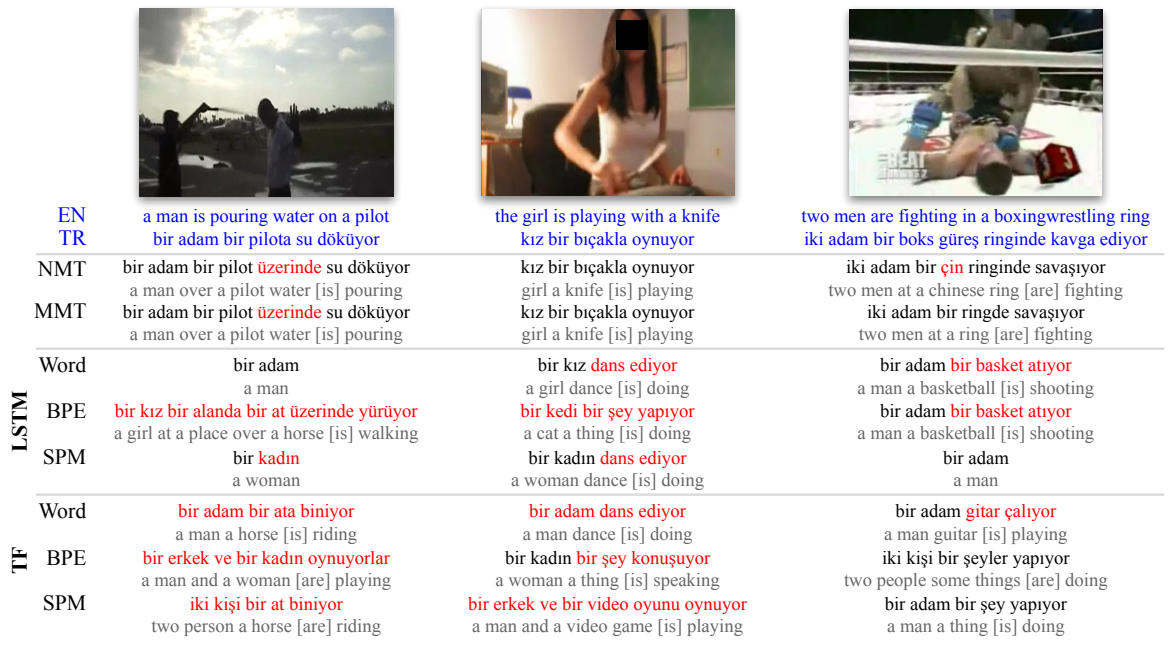

Fig. 7 Video captioning and machine translation results on MSVD test set: TF (Transformer) and LSTM refer to the video captioning outputs, MMT system is the enc-dec initialisation variant from Table 4 . The ground-truth captions, English gloss translations and incorrect generations are given in blue, gray and red, respectively. The examples (A) to (C) and (D) to (F) depict good and bad outputs, respectively.

\section{Conclusion}

In this paper we introduced and described a new large-scale video description dataset called MSVD-Turkish, which was constructed by carefully translating original English descriptions of MSVD dataset (Chen and Dolan, 2011) to Turkish. Our dataset will allow research on novel video captioning models for Turkish, a highly inflected and agglutinative language, as well as on 
multilingual video captioning approaches, including those based on translation. Additionally, as our the Turkish descriptions are direct translations of English descriptions, the dataset can be used for research in novel approaches to multimodal machine translation.

We also provided baselines using popular neural models based on recurrent neural networks and Transformer architectures. For which of these neural architectures, we analysed the use of word segmentation approaches such as BPE and SPM and demonstrated how they help both description generation and machine translation. We hope that our dataset will serve as a good resource for future efforts on multilingual, multimodal language generation. As a future work, it would be interesting to study the intrinsic annotation biases or linguistic differences between English and Turkish descriptions in MSVD and MSVD-Turkish datasets.

Acknowledgements This work was supported in part by TUBA GEBIP fellowship awarded to E. Erdem, and the MMVC project funded by TUBITAK and the British Council via the Newton Fund Institutional Links grant programme (grant ID 219E054 and 352343575). Lucia Specia, Pranava Madhyastha and Ozan Caglayan also received support from MultiMT (H2020 ERC Starting Grant No. 678017).

\section{References}

Aafaq N, Mian A, Liu W, Gilani SZ, Shah M (2019) Video description: A survey of methods, datasets and evaluation metrics and description. ACM Comput Surv 52(6)

Akin AA, Akin MD (2007) Zemberek, an open source nlp framework for turkic languages

Bahdanau D, Cho K, Bengio Y (2015) Neural machine translation by jointly learning to align and translate. Proc International Conference on Learning Representations (ICLR) URL http: //arxiv .org/pdf/1409.0473

Barbu A, Bridge A, Burchill Z, Coroian D, Dickinson S, Fidler S, Michaux A, Mussman S, Narayanaswamy S, Salvi D, Schmidt L, Shangguan J, Siskind JM, Waggoner J, Wang S, Wei J, Yin Y, Zhang Z (2012) Video in sentences out. Proc 28th Conference on Uncertainty in Artificial Intelligence (UAI2012) URL http://arxiv.org/abs/1204.2742 1204.2742

Barrault L, Bougares F, Specia L, Lala C, Elliott D, Frank S (2018) Findings of the third shared task on multimodal machine translation. In: Proc. Third Conference on Machine Translation, Volume 2: Shared Task Papers, Association for Computational Linguistics, Belgium, Brussels, pp 308-327, URL http://www. aclweb.org/anthology/W18-6402

Caglayan O, Aransa W, Wang Y, Masana M, García-Martínez M, Bougares F, Barrault L, van de Weijer J (2016a) Does multimodality help human and machine for translation and image captioning? In: Proc. First Conference on Machine Translation, Association for Computational Linguistics, Berlin, Germany, pp 627-633, URL http://www.aclweb. org/anthology/W/W16/W16-2358

Caglayan O, Barrault L, Bougares F (2016b) Multimodal attention for neural machine translation. Computing Research Repository arXiv:1609.03976, URL http://arxiv.org/pdf/ 1609.03976

Caglayan O, Aransa W, Bardet A, García-Martínez M, Bougares F, Barrault L, Masana M, Herranz L, van de Weijer J (2017a) LIUM-CVC submissions for WMT17 multimodal translation task. In: Proc. Second Conference on Machine Translation, Volume 2: Shared Task Papers, Association for Computational Linguistics, Copenhagen, Denmark, pp 432439, URL http://www . aclweb.org/anthology/W17-4746

Caglayan O, García-Martínez M, Bardet A, Aransa W, Bougares F, Barrault L (2017b) NMTPY: A flexible toolkit for advanced neural machine translation systems. Prague 
Bull Math Linguistics 109:15-28, DOI 10.1515/pralin-2017-0035, URL https://ufal. mff.cuni.cz/pbml/109/art-caglayan-et-al.pdf

Calixto I, Liu Q (2017) Incorporating global visual features into attention-based neural machine translation. In: Proc. Conference on Empirical Methods in Natural Language Processing (EMNLP), Association for Computational Linguistics, Copenhagen, Denmark, pp 992-1003, URL https://www.aclweb.org/anthology/D17-1105

Calixto I, Elliott D, Frank S (2016) DCU-UvA multimodal MT system report. In: Proceedings of the First Conference on Machine Translation, Association for Computational Linguistics, Berlin, Germany, pp 634-638, URL http://www.aclweb.org/anthology/W/ W16/W16-2359

Chen D, Dolan W (2011) Collecting highly parallel data for paraphrase evaluation. In: Proceedings of the 49th Annual Meeting of the Association for Computational Linguistics (ACL): Human Language Technologies, Association for Computational Linguistics, Portland, Oregon, USA, pp 190-200, URL https://www.aclweb.org/anthology/P11-1020

Chen Y, Wang S, Zhang W, Huang Q (2018) Less is more: Picking informative frames for video captioning. CoRR abs/1803.01457, URL http://arxiv.org/abs/1803.01457. 1803.01457

Cho K, van Merrienboer B, Gulcehre C, Bahdanau D, Bougares F, Schwenk H, Bengio Y (2014) Learning phrase representations using rnn encoder-decoder for statistical machine translation. In: Proc. Conference on Empirical Methods in Natural Language Processing (EMNLP), Association for Computational Linguistics, Doha, Qatar, pp 1724-1734, URL http://www.aclweb.org/anthology/D14-1179

Das P, Xu C, Doell R, Corso J (2013) A thousand frames in just a few words: Lingual description of videos through latent topics and sparse object stitching. In: Proc. IEEE International Conference on Computer Vision and Pattern Recognition (CVPR), pp 26342641, DOI 10.1109/CVPR.2013.340

Denkowski M, Lavie A (2014) Meteor universal: Language specific translation evaluation for any target language. In: Proc. 9th Workshop on Statistical Machine Translation, Association for Computational Linguistics, pp 376-380, DOI 10.3115/v1/W14-3348, URL http://aclweb.org/anthology/W14-3348

Donahue J, Hendricks LA, Guadarrama S, Rohrbach M, Venugopalan S, Saenko K, Darrell $\mathrm{T}$ (2015) Long-term recurrent convolutional networks for visual recognition and description. Proc IEEE Conference on Computer Vision and Pattern Recognition (CVPR) abs/1411.4389, URL http://arxiv.org/abs/1411.4389, 1411.4389

Elliott D, Kádár À (2017) Imagination improves multimodal translation. In: Proc. 8th International Joint Conference on Natural Language Processing (IJCNLP) (Volume 1: Long Papers), Asian Federation of Natural Language Processing, pp 130-141, URL http://aclweb.org/anthology/I17-1014

Elliott D, Frank S, Sima'an K, Specia L (2016) Multi30k: Multilingual english-german image descriptions. In: Proc. 5th Workshop on Vision and Language, Association for Computational Linguistics, Berlin, Germany, pp 70-74, URL http://anthology.aclweb.org/ W16-3210

Elliott D, Frank S, Barrault L, Bougares F, Specia L (2017) Findings of the second shared task on multimodal machine translation and multilingual image description. In: Proc. 2nd Conference on Machine Translation, Volume 2: Shared Task Papers, Association for Computational Linguistics, Copenhagen, Denmark, pp 215-233, URL http://www . aclweb.org/anthology/W17-4718

Gella S, Lewis M, Rohrbach M (2018) A dataset for telling the stories of social media videos. In: Proc. Conference on Empirical Methods in Natural Language Processing (EMNLP), Association for Computational Linguistics, Brussels, Belgium, pp 968-974, DOI 10.18653/ v1/D18-1117, URL https://www.aclweb.org/anthology/D18-1117

Guadarrama S, Krishnamoorthy N, Malkarnenkar G, Venugopalan S, Mooney R, Darrell T, Saenko K (2013) Youtube2text: Recognizing and describing arbitrary activities using semantic hierarchies and zero-shot recognition. In: Proc. IEEE International Conference on Computer Vision (ICCV), Sydney, Australia, pp 2712-2719, URL http://www.cs . utexas.edu/users/ai-labpub-view.php?PubID=127409 
Hakeem A, Sheikh Y, Shah M (2004) case c $^{e}$ A hierarchical event representation for the analysis of videos. In: Proc. Association for the Advancement of Artificial Intelligence (AAAI), pp 263-268

Hanckmann P, Schutte K, Burghouts GJ (2012) Automated textual descriptions for a wide range of video events with 48 human actions. In: Proc. European Conference on Computer Vision (ECCV)

He K, Xiangyu Z, Shaoqing R, Sun J (2016) Deep residual learning for image recognition. In: Proc. IEEE Conference on Computer Vision and Pattern Recognition (CVPR), pp 770-778, DOI 10.1109/CVPR.2016.90

Hochreiter S, Schmidhuber J (1997) Long Short-term Memory. Neural computation 9(8):1735-1780

Huang PY, Liu F, Shiang SR, Oh J, Dyer C (2016) Attention-based multimodal neural machine translation. In: Proc. First Conference on Machine Translation, Association for Computational Linguistics, Berlin, Germany, pp 639-645, URL http://www.aclweb.org/ anthology/W/W16/W16-2360

Inan H, Khosravi K, Socher R (2016) Tying word vectors and word classifiers: A loss framework for language modeling. CoRR arXiv:1611.01462, URL http://arxiv .org/pdf/1611. 01462

Kingma D, Ba J (2014) Adam: A method for stochastic optimization. CoRR arXiv:1412.6980, URL http://arxiv.org/pdf/1412.6980

Kojima A, Tamura T, Fukunaga K (2012) Natural language description of human activities from video images based on concept hierarchy of actions. International Journal of Computer Vision (IJCV) 50(2):171-184

Krishna R, Hata K, Ren F, Li F, Niebles JC (2017) Dense-captioning events in videos. CoRR abs/1705.00754, URL http://arxiv.org/abs/1705.00754, 1705.00754

Krishnamoorthy N, Malkarnenkar G, Mooney R, Saenko K, Guadarrama S (2013) Generating natural-language video descriptions using text-mined knowledge. In: Proc. Annual Conference of the North American Chapter of the Association for Computational Linguistics (NAACL): Human Language Technologies, Association for Computational Linguistics, Atlanta, Georgia, pp 10-19, URL https://www.aclweb.org/anthology/W13-1302

Kudo T, Richardson J (2018) Sentencepiece: A simple and language independent subword tokenizer and detokenizer for neural text processing. In: Proc. Conference on Empirical Methods in Natural Language Processing (EMNLP): System Demonstrations, Association for Computational Linguistics, Brussels, Belgium, pp 66-71, URL http://www .aclweb . org/anthology/D18-2012

Li Y, Song Y, Cao L, Tetreault JR, Goldberg L, Jaimes A, Luo J (2016) TGIF: A new dataset and benchmark on animated GIF description. Proc IEEE Conference on Computer Vision and Pattern Recognition (CVPR) URL http://arxiv.org/abs/1604.02748, 1604.02748

Libovický J, Helcl J (2017) Attention strategies for multi-source sequence-to-sequence learning. In: Proc. 55th Annual Meeting of the Association for Computational Linguistics (ACL) (Volume 2: Short Papers), Association for Computational Linguistics, pp 196202, DOI 10.18653/v1/P17-2031, URL http://aclweb.org/anthology/P17-2031

Lin CY (2004) ROUGE: A package for automatic evaluation of summaries. In: Proc. Annual Meeting of the Association for Computational Linguistics (ACL), pp 74-81

Lin TY, Maire M, Belongie S, Hays J, Perona P, Ramanan D, Dollár P, Zitnick CL (2014) Microsoft COCO: Common objects in context. In: Proc. European Conference on Computer Vision (ECCV), Springer, pp 740-755

Loper E, Bird S (2002) NLTK: The natural language toolkit. In: Proceedings of the ACL-02 Workshop on Effective Tools and Methodologies for Teaching Natural Language Processing and Computational Linguistics, Association for Computational Linguistics, Philadelphia, Pennsylvania, USA, pp 63-70, DOI 10.3115/1118108.1118117, URL https://www.aclweb.org/anthology/W02-0109

Ott M, Edunov S, Baevski A, Fan A, Gross S, Ng N, Grangier D, Auli M (2019) fairseq: A Fast, Extensible Toolkit for Sequence Modeling. In: Proc. Conference of the North American Chapter of the Association for Computational Linguistics (NAACL): Human Language Technologies

Papineni K, Roukos S, Ward T, Zhu WJ (2002) Bleu: A method for automatic evaluation of machine translation. In: Proc. 40th Annual Meeting on Association for Computational 
Linguistics, Association for Computational Linguistics, Stroudsburg, PA, USA, pp 311318, DOI 10.3115/1073083.1073135, URL http://dx.doi.org/10.3115/1073083.1073135

Plummer BA, Wang L, Cervantes CM, Caicedo JC, Hockenmaier J, Lazebnik S (2015) Flickr30k entities: Collecting region-to-phrase correspondences for richer image-tosentence models. In: Proc. IEEE International Conference on Computer Vision (ICCV), pp 2641-2649, DOI 10.1109/ICCV.2015.303

Press O, Wolf L (2017) Using the output embedding to improve language models. Proc 15th Conference of the European Chapter of the Association for Computational Linguistics URL http://arxiv.org/abs/1608.05859, 1608.05859

Regneri M, Rohrbach M, Wetzel D, Thater S, Schiele B, Pinkal M (2013) Grounding action descriptions in videos. Transactions of the Association for Computational Linguistics 1:25-36, URL https://www . aclweb.org/anthology/Q13-1003

Rohrbach A, Rohrbach M, Tandon N, Schiele B (2015) A dataset for movie description. In: Proc. IEEE International Conference on Computer Vision and Pattern Recognition (CVPR), pp 3202-3212, DOI 10.1109/CVPR.2015.7298940

Rohrbach A, Torabi A, Rohrbach M, Tandon N, Pal C, Larochelle H, Courville A, Schiele B (2017) Movie description. Int J Comput Vision 123(1):94-120, DOI 10.1007/ s11263-016-0987-1, URL https://doi.org/10.1007/s11263-016-0987-1

Rohrbach M, Amin S, Andriluka M, Schiele B (2012) A database for fine grained activity detection of cooking activities. In: Proc. IEEE International Conference on Computer Vision and Pattern Recognition (CVPR), IEEE Computer Society, pp 1194-1201

Rohrbach M, Qiu W, Titov I, Thater S, Pinkal M, Schiele B (2013) Translating video content to natural language descriptions. In: Proc. IEEE International Conference on Computer Vision (ICCV), IEEE Computer Society, pp 433-440

Senina A, Rohrbach M, Qiu W, Friedrich A, Amin S, Andriluka M, Pinkal M, Schiele B (2014) Coherent multi-sentence video description with variable level of detail. CoRR abs/1403.6173, URL http://arxiv.org/abs/1403.6173 1403.6173

Sennrich R, Haddow B, Birch A (2016) Neural machine translation of rare words with subword units. In: Proc. 54th Annual Meeting of the Association for Computational Linguistics (Volume 1: Long Papers), Association for Computational Linguistics, Berlin, Germany, pp 1715-1725, URL http://www.aclweb.org/anthology/P16-1162

Sennrich R, Firat O, Cho K, Birch-Mayne A, Haddow B, Hitschler J, Junczys-Dowmunt M, Läubli S, Miceli Barone A, Mokry J, Nadejde M (2017) Nematus: a Toolkit for Neural Machine Translation, Association for Computational Linguistics (ACL), pp 65-68

Sigurdsson GA, Varol G, Wang X, Farhadi A, Laptev I, Gupta A (2016) Hollywood in homes: Crowdsourcing data collection for activity understanding. CoRR abs/1604.01753, URL http://arxiv.org/abs/1604.01753, 1604.01753

Simonyan K, Zisserman A (2014) Very deep convolutional networks for large-scale image recognition. URL http://arxiv.org/abs/1409.1556 cite arxiv:1409.1556

Specia L, Frank S, Sima'an K, Elliott D (2016) A shared task on multimodal machine translation and crosslingual image description. In: Proc. of the First Conference on Machine Translation, Association for Computational Linguistics, Berlin, Germany, pp 543-553, URL http://www . aclweb.org/anthology/W/W16/W16-2346

Srivastava N, Hinton G, Krizhevsky A, Sutskever I, Salakhutdinov R (2014) Dropout: A simple way to prevent neural networks from overfitting. The Journal of Machine Learning Research 15(1):1929-1958, URL http://dl . acm.org/citation. cfm?id=2627435.2670313

Srivastava N, Mansimov E, Salakhutdinov R (2015) Unsupervised learning of video representations using lstms. CoRR abs/1502.04681, URL http://arxiv.org/abs/1502.04681 1502.04681

Sulubacak U, Caglayan O, Grönroos SA, Rouhe A, Elliott D, Specia L, Tiedemann J (2019) Multimodal Machine Translation through Visuals and Speech. arXiv preprint arXiv: 191112798

Sutskever I, Vinyals O, Le QV (2014) Sequence to sequence learning with neural networks. In: Proc. 27th International Conference on Neural Information Processing Systems(NIPS), MIT Press, Cambridge, MA, USA, pp 3104-3112, URL http://dl.acm.org/citation. cfm?id=2969033.2969173

Thomason J, Venugopalan S, Guadarrama S, Saenko K, Mooney R (2014) Integrating language and vision to generate natural language descriptions of videos in the wild. In: Proc. 
International Conference on Computational Linguistics (COLING): Technical Papers, pp 1218-1227, URL https://www . aclweb.org/anthology/C14-1115

Torabi A, Pal CJ, Larochelle H, Courville AC (2015) Using descriptive video services to create a large data source for video annotation research. CoRR abs/1503.01070, URL http://arxiv.org/abs/1503.01070 1503.01070

Unal ME, Citamak B, Yagcioglu S, Erdem A, Erdem E, Cinbis NI, Cakici R (2016) TasvirEt: A benchmark dataset for automatic Turkish description generation from images. In: Proc. 24th Signal Processing and Communication Application Conference (SIU), pp 1977-1980, DOI 10.1109/SIU.2016.7496155

Vaswani A, Shazeer N, Parmar N, Uszkoreit J, Jones L, Gomez AN, Kaiser Lu, Polosukhin I (2017) Attention is all you need. In: Guyon I, Luxburg UV, Bengio S, Wallach H, Fergus R, Vishwanathan S, Garnett R (eds) Advances in Neural Information Processing Systems 30, Curran Associates, Inc., pp 5998-6008, URL http://papers.nips.cc/paper/ 7181-attention-is-all-you-need.pdf

Vaswani A, Bengio S, Brevdo E, Chollet F, Gomez AN, Gouws S, Jones L, Kaiser L, Kalchbrenner N, Parmar N, Sepassi R, Shazeer N, Uszkoreit J (2018) Tensor2tensor for neural machine translation. CoRR abs/1803.07416, URL http://arxiv.org/abs/1803.07416

Vedantam R, Lawrence Zitnick C, Parikh D (2015) Cider: Consensus-based image description evaluation. In: Proc. IEEE International Conference on Computer Vision and Pattern Recognition (CVPR), pp 4566-4575

Venugopalan S, Rohrbach M, Donahue J, Mooney R, Darrell T, Saenko K (2015) Sequence to Sequence-Video to Text. In: Proc. IEEE International Conference on Computer Vision (ICCV)

Wang X, Chen W, Wu J, Wang Y, Wang WY (2018) Video captioning via hierarchical reinforcement learning. Proc IEEE International Conference on Computer Vision and Pattern Recognition (CVPR) URL http://arxiv.org/abs/1711.11135, 1711.11135

Wang X, Wu J, Chen J, Li L, Wang YF, Wang WY (2019) Vatex: A large-scale, highquality multilingual dataset for video-and-language research. In: Proc. IEEE International Conference on Computer Vision (ICCV)

Xu J, Mei T, Yao T, Rui Y (2016) MSR-VTT: A Large Video Description Dataset for Bridging Video and Language. In: Proc. IEEE International Conference on Computer Vision and Pattern Recognition (CVPR), URL https://www.microsoft.com/en-us/research/publication/ msr-vtt-a-large-video-description-dataset-for-bridging-video-and-language/

Xu K, Ba J, Kiros R, Cho K, Courville A, Salakhudinov R, Zemel R, Bengio Y (2015) Show, attend and tell: Neural image caption generation with visual attention. In: Proc. 32nd International Conference on Machine Learning (ICML), JMLR Workshop and Conference Proceedings, pp 2048-2057, URL http://jmlr.org/proceedings/papers/v37/xuc15.pdf

Yao L, Torabi A, Cho K, Ballas N, Pal C, Larochelle H, Courville A (2015) Describing videos by exploiting temporal structure. In: Proc. IEEE International Conference on Computer Vision (ICCV)

Yoshikawa Y, Shigeto Y, Takeuchi A (2017) STAIR captions: Constructing a large-scale japanese image caption dataset. Proc 55th Annual Meeting of the Association for Computational Linguistics URL http://arxiv.org/abs/1705.00823, 1705.00823

Yu H, Wang J, Huang Z, Yang Y, Xu W (2016) Video paragraph captioning using hierarchical recurrent neural networks. Proc IEEE Conference on Computer Vision and Pattern Recognition (CVPR) URL http://arxiv.org/abs/1510.07712, 1510.07712

Zeng K, Chen T, Niebles JC, Sun M (2016) Title generation for user generated videos. Proc IEEE Conference on Computer Vision and Pattern Recognition (CVPR) URL http: //arxiv.org/abs/1608.07068, 1608.07068

Zhou L, Xu C, Corso JJ (2017) Procnets: Learning to segment procedures in untrimmed and unconstrained videos. CoRR abs/1703.09788, URL http://arxiv.org/abs/1703.09788 1703.09788

Zhou L, Kalantidis Y, Chen X, Corso JJ, Rohrbach M (2019) Grounded video description. Proc IEEE Conference on Computer Vision and Pattern Recognition (CVPR) URL http: //arxiv.org/abs/1812.06587, 1812.06587 